\title{
More about 中文写作
}

During the forty years that I have taught Chinese at the college level, I have found that textbooks generally fail to provide much in the way of level-appropriate models for the various kinds of writing that I ask my students to produce and that are most useful for them in their learning journey, especially at that critical intermediate level. For example, textbooks do not provide detailed explanations concerning the format for writing a letter. Which are the customary greetings and closing? How should a letter be formatted? Rarely do textbooks include examples and focused help in producing the many essays or correspondences that we ask our students to write on different aspects of their everyday lives.

My Chinese wife and I are both longtime teachers of Chinese at Calvin University. We have discovered that students often lack an understanding of how to structure the piece of writing they are being asked to produce. They also lack sufficient vocabulary needed to write in any depth about their own personal experiences, limited as they are to the vocabulary provided in standard textbooks. In addition, a significant number of students make considerable mistakes in grammar, including the major issues of word choice and word order. This book provides models our students desperately need for all types of essays, e-mails, letters, and narrative writing at the intermediate level.

For each writing sample, we provide sections called "Words and Phrases" and "Notes." For the Level A examples, the "Words and Phrases" sections do not generally include the basic vocabulary that is taught in textbooks. Instead, we focus on building and expanding vocabulary with topic-specific words and phrases that can be added to the basic vocabulary and then adapted by learners in their own writing. Similarly, for Level B examples, we generally do not include vocabulary found in standard textbooks for middle- and high-intermediate levels. However, if words or grammatical patterns are particularly important for a text topic or genre, we include them as extra support for learners.

The useful writing samples are the base for our MPG approach to learning to write. It is our hope that learners will also use these models for additional practice in reading comprehension and text analysis. Almost all the essays are based on essays written by our college students. The intent is to provide texts that reflect the genuine life and feelings of typical students today. The "Words and Phrases" sections and models for correspondence used in this book are based on usage in mainland China rather than Taiwan or Hong Kong, where the language in such instances is much more formal and employs many more terms from classical Chinese.

Our main goal in this book is to help Chinese learners, especially at the intermediate level, to express themselves in writing in correct Chinese on common everyday topics and for common social purposes. By providing sample texts with vocabulary, tips, and strategies for success in using those texts as models, we hope to enable both learners and their teachers to enjoy the process of developing solid writing skills in Chinese. 